\title{
First Tau PET Tracer Approved: Toward Accurate In Vivo Diagnosis of Alzheimer Disease
}

\author{
Henryk Barthel \\ Department of Nuclear Medicine, University of Leipzig, Leipzig, Germany
}

$\mathbf{O}$ n May 28, the Food and Drug Administration approved ${ }^{18} \mathrm{~F}$ flortaucipir, also known as AV1451, under the trademark name Tauvid (Avid Radiopharmaceuticals), for estimation of the density and distribution of aggregated tau neurofibrillary tangles in adults with cognitive impairment who are being evaluated for Alzheimer disease (AD). As such, ${ }^{18} \mathrm{~F}$-flortaucipir is the first approved tau PET tracer. This is a great achievement and a major step forward in our mission to improve AD diagnosis.

$\mathrm{AD}$ is histopathologically characterized by the presence of $\beta$-amyloid plaques and neurofibrillary tangles in the brain. To detect amyloid pathology in vivo, several PET tracers are approved. Establishment of cerebral tau pathology, however, has been possible so far only by experimental PET tracers or after death via histopathologic examination. We now have the opportunity to shift this important event into the clinic-that is, to a time point at which the tau diagnosis might influence patient management.

${ }^{18} \mathrm{~F}$-flortaucipir was developed as ${ }^{18} \mathrm{~F}-\mathrm{T} 807$ and successfully brought to human use in 2012 by Hartmuth Kolb's group at Siemens Healthcare (1). In 2013, the rights were acquired by Avid. The recent Food and Drug Administration approval of this tracer was based on positive tracer safety data as obtained in more than 1,900 subjects, and on a successful pivotal phase 3 program. In the latter, in vivo PET imaging was compared in 2 trials, intraindividually, with postmortem histopathology, and favorable results were recently published in JAMA Neurology (2). The editorial by Mattay et al. (3) in this issue of The Journal of Nuclear Medicine provides more insight into the Food and Drug Administration's perspective on the filed data and the approval. I am taking the chance here to add my perspective as a brain PET scientist to this new, exciting development.

It is important to underline that ${ }^{18} \mathrm{~F}$-flortaucipir should not be clinically used to image tauopathies other than AD. According to Avid, for clinical use in suspected AD patients the PET images will be evaluated visually in a binary manner. In the above histopathology trial, a positive PET scan was associated with an advanced (so-called B3) stage at which the tau pathology is spread throughout the neocortex. Of interest, the fact that those patients were also $\beta$-amyloid plaque-positive on histopathology suggests that a positive ${ }^{18} \mathrm{~F}$-flortaucipir scan has the potential to (directly

Received Jun. 24, 2020; revision accepted Jun. 30, 2020.

For correspondence or reprints contact: Henryk Barthel, Leipzig Univeristy, Liebigstrasse 18, Leipzig, 04103 Germany.

E-mail: henryk.barthel@medizin.uni-leipzig.de

COPYRIGHT (C) 2020 by the Society of Nuclear Medicine and Molecular Imaging. DOI: 10.2967/jnumed.120.252411 and indirectly) provide a comprehensive AD diagnosis. This concept is exciting and should be further tested in prospective trials. We will soon also need to establish recommendations on the clinical scenarios that are appropriate for tau versus amyloid PET imaging; the well-established appropriate-use criteria for amyloid imaging $(4,5)$ certainly represent a valuable starting point for this task.

One drawback is that a negative ${ }^{18} \mathrm{~F}$-flortaucipir scan does not exclude earlier (B1 to B2) tau pathology stages in AD in which neurofibrillary tangles are only present in deeper brain regions. This limited ability to detect earlier tau stages is probably caused by off-target, non-tau-related binding of the tracer, causing difficulties in PET image interpretation in deeper brain structures. We will need to come to an agreement on how to further evaluate such PET-negative cases. Regardless, there is great potential for ${ }^{18}$ F-flortaucipir to make a difference, especially in the field of differential dementia diagnosis. In the dementia stage, at which cognitive impairment is so advanced that it affects the activities of daily living, a negative ${ }^{18} \mathrm{~F}$-flortaucipir scan will be able to exclude $\mathrm{AD}$ and point to other, non- $\beta$-amyloid or tau-related, neurodegenerative disorders with high accuracy (6). It will be exciting to see how this concept will enter clinical routine. According to Avid, the tracer will be available in the fall of 2020 on a limited basis, but without being covered under the Medicare benefit. It will be another task for our community and the payers to discuss whether, and how, wider access to and coverage for this novel tool should be pursued.

The real work has just started in bringing tau PET imaging into the clinic, and more research is necessary to answer questions that exist both for the now-approved ${ }^{18} \mathrm{~F}$-flortaucipir and for the concept of tau PET imaging in general. The most burning questions in this regard are: What is the optimal visual read algorithm to evaluate ${ }^{18}$ F-flortaucipir images? Will image quantification be integrated in the respective PET image interpretation? Will ${ }^{18} \mathrm{~F}$-flortaucipir also be of clinical use in the future for $\mathrm{AD}$ progression estimation? Is ${ }^{18} \mathrm{~F}$-flortaucipir the optimal tau tracer for research applications such as proof of drug target engagement or imaging of tau aggregates in chronic traumatic encephalopathy? Will we need more advanced tau tracers to image so-called 4R tauopathies such as corticobasal degeneration or progressive supranuclear palsy? Will novel tau tracers be able to cover earlier tau stages in AD? The opportunity to find answers to these and other related questions will certainly excite many academic and commercial brain PET researchers in the next years to come.

On a practical note of caution for the application of the nowapproved tau tracer ${ }^{18} \mathrm{~F}$-flortaucipir, strict adherence to the recommended scan start time ( 80 min after injection) and scan duration 
(20 min) is definitely recommended. This recommendation is based on the fact that the tracer does not reach apparent steady state over this time range (1). Following these rules will improve the comparability of the resulting static images or semiquantitative data between subjects and over time. In an ideal world where longer imaging times are possible, dynamic imaging allowing for full quantification of the tracer binding via kinetic modeling is recommended (7).

The approval of ${ }^{18} \mathrm{~F}$-flortaucipir as the first tau PET tracer is a great achievement and an important milestone. We applaud the sponsor and the involved researchers and patients whose skills, effort, and enthusiasm have provided much motivation to the entire brain imaging community on its joint goal of improving dementia diagnosis and care.

\section{DISCLOSURE}

No potential conflict of interest relevant to this article was reported.

\section{REFERENCES}

1. Chien DT, Bahri S, Szardenings AK, et al. Early clinical PET imaging results with the novel PHF-tau radioligand [F-18]-T807. J Alzheimers Dis. 2013;34:457-468.

2. Fleisher AS, Pontecorvo MJ, Devous MD Sr, et al. Positron emission tomography imaging with $\left[{ }^{18} \mathrm{~F}\right]$ flortaucipir and postmortem assessment of Alzheimer disease neuropathologic changes. JAMA Neurol. April 26, 2020 [Epub ahead of print].

3. Mattay VS, Fotenos AF, Ganley CJ, Marzella L. Brain tau imaging: Food and Drug Administration approval of ${ }^{18}$ F-flortaucipir injection. $J$ Nucl Med. 2020;61: 1411-1412.

4. Johnson KA, Minoshima S, Bohnen NI, et al. Appropriate use criteria for amyloid PET: a report of the Amyloid Imaging Task Force, the Society of Nuclear Medicine and Molecular Imaging, and the Alzheimer's Association. J Nucl Med.2013;54: 476-490.

5. Johnson KA, Minoshima S, Bohnen NI, et al. Update on appropriate use criteria for amyloid PET imaging: dementia experts, mild cognitive impairment, and education. J Nucl Med. 2013;54:1011-1013.

6. Ossenkoppele R, Rabinovici GD, Smith R, et al. Discriminative Accuracy of $\left[{ }^{18} \mathrm{~F}\right]$ flortaucipir positron emission tomography for Alzheimer disease vs other neurodegenerative disorders. JAMA. 2018;320:1151-1162.

7. Barthel H, Seibyl J, Lammertsma AA, Villemagne VL, Sabri O. Exploiting the full potential of $\beta$-amyloid and tau PET imaging for drug efficacy testing. $\mathrm{J} \mathrm{Nucl}$ Med. 2020;61:1105-1106. 